\title{
Determinants and predictors for the long-term disease burden of intracranial meningioma patients
}

\author{
Amir H. Zamanipoor Najafabadi ${ }^{1,2,3}$ - Pim B. van der Meer ${ }^{3}$. Florien W. Boele ${ }^{4,5} \cdot$ Martin J. B. Taphoorn $^{3,7}$. \\ Martin Klein ${ }^{8}$. Saskia M. Peerdeman ${ }^{6} \cdot$ Wouter R. van Furth $^{1,2} \cdot$ Linda Dirven $^{3,7}$
}

Received: 23 August 2020 / Accepted: 12 October 2020 / Published online: 19 October 2020

(c) The Author(s) 2020

\begin{abstract}
Introduction Meningioma is a heterogeneous disease and patients may suffer from long-term tumor- and treatment-related sequelae. To help identify patients at risk for these late effects, we first assessed variables associated with impaired longterm health-related quality of life (HRQoL) and impaired neurocognitive function on group level (i.e. determinants). Next, prediction models were developed to predict the risk for long-term neurocognitive or HRQoL impairment on individual patient-level.

Methods Secondary data analysis of a cross-sectional multicenter study with intracranial WHO grade I/II meningioma patients, in which HRQoL (Short-Form 36) and neurocognitive functioning (standardized test battery) were assessed. Multivariable regression models were used to assess determinants for these outcomes corrected for confounders, and to build prediction models, evaluated with C-statistics.

Results Data from 190 patients were analyzed (median 9 years after intervention). Main determinants for poor HRQoL or impaired neurocognitive function were patients' sociodemographic characteristics, surgical complications, reoperation, radiotherapy, presence of edema, and a larger tumor diameter on last MRI. Prediction models with a moderate/good ability to discriminate between individual patients with and without impaired HRQoL (C-statistic 0.73, 95\% CI 0.65 to 0.81 ) and neurocognitive function (C-statistic $0.78,95 \%$ CI 0.70 to 0.85 ) were built. Not all predictors (e.g. tumor location) within these models were also determinants.

Conclusions The identified determinants help clinicians to better understand long-term meningioma disease burden. Prediction models can help early identification of individual patients at risk for long-term neurocognitive or HRQoL impairment, facilitating tailored provision of information and allocation of scarce supportive care services to those most likely to benefit.
\end{abstract}

Keywords Meningioma $\cdot$ Health-related quality of life $\cdot$ Neurocognitive functioning $\cdot$ Predictors $\cdot$ Determinants $\cdot$ Risk factors

Members of Dutch Meningioma Consortium are provided in Acknowledgements section.

Electronic supplementary material The online version of this article (https://doi.org/10.1007/s11060-020-03650-1) contains supplementary material, which is available to authorized users.

Amir H. Zamanipoor Najafabadi amir@lumc.nl

Extended author information available on the last page of the article

\section{Introduction}

Although over $95 \%$ of meningioma patients have a nonmalignant WHO grade I or II tumor [1], these patients still suffer from a clinically relevant disease burden, even after tumor resection, which can persist over time [2-6]. Compared with controls, meningioma patients report on average worse health-related quality of life (HRQoL) up to 9 years after surgery [3,4]. Approximately $40 \%$ of patients have neurocognitive impairments, although these impairments are often not considered clinically meaningful [5-7]. However, not all meningioma patients have poor outcomes and it is currently unclear which factors are related to the long-term disease burden, while it might 
help to better understand the disease burden in meningioma patients. In the clinical setting, early identification of patients at high risk for a long-term disease burden facilitates timely provision of information and rehabilitation, and allocation of scarce supportive care services to those most likely to obtain benefit.

A limited number of published studies have reported a variety of variables associated with increased meningioma disease burden in the first years after treatment, primarily focusing on sociodemographic (e.g. higher age and lower educational level), tumor (e.g. larger tumor diameter and higher WHO grade) and treatment characteristics (e.g. higher Simpson grade and receiving radiotherapy) [3, 7]. However, there are no published studies on the possible factors associated with the long-term disease burden ( $\geq 5$ years). This distinction is important as patients might suffer from different issues during the treatment phase, then they do on the longer term (i.e. survivorship issues). First, some aspects of treatment toxicity only become apparent on the long-term, e.g. neurocognitive impairments caused by radiotherapy [2, 8-11]. Second, patients learn to adapt to the disease-related symptoms and change their coping strategies over time, influencing patients' perception of their disease burden [2, 12]. Finally, on the long-term patients might face growth of tumor remnant or recurrence of disease, sometimes requiring intervention [13].

A methodological limitation of most published studies determining associations between certain risk factors and outcomes is the lack of distinction between determinants and predictors [14]. A determinant is an individual variable that on group-level is independently associated with the outcome of interest, corrected for confounding (e.g. the association between sex or tumor location with the long-term disease burden). Prediction models on the other hand use multiple variables together (i.e. patient, tumor and treatment characteristics) to predict for an individual patient the risk to develop a certain outcome of interest. Although both reflect patients' future outcomes, determinants are variables with an assumed causal relationship to the outcome of interest (e.g. postoperative complications may have a negative impact on a patient's long-term HRQoL), while predictors are solely used to predict the outcome of interest (e.g. hospitalization length may be predictive for diminished HRQoL on the long-term), without assuming causality.

We aimed to assess in meningioma patients determinants for the long-term disease burden, defined as impaired HRQoL and neurocognitive function at a median of 9 years after the last intervention. Furthermore, we have built prediction models to identify individual patients with a high risk around the time of intervention to suffer from a longterm impairment in HRQoL or neurocognitive function.

\section{Methods}

\section{Participants}

This is a secondary analysis of a multicenter cross-sectional study, assessing the long-term disease burden in meningioma patients [15]. Consecutive meningioma patients were recruited from the neurology, neurosurgery and radiation oncology outpatient clinics of two academic hospitals and one large non-academic teaching hospital between July 2016 and April 2019. Patients were eligible if the end of their anti-tumor treatment was at least 5 years prior to recruitment, or in case of active MRI surveillance, at least 5 years after diagnosis. Furthermore, patients had to be 18 years or older; with a histologically confirmed WHO grade I or II meningioma in case of surgery, and an MRI-based clinically suspected meningioma in case of radiotherapy only or active MRI surveillance. Exclusion criteria for study participation were history of whole brain radiotherapy, diagnosis with a neurodegenerative disease (including neurofibromatosis type II), or patients not proficient in the Dutch language.

\section{Procedures}

Information on tumor and treatment was obtained from patient's charts, and sociodemographic information was obtained at the beginning of the assessments (questionnaires and neurocognitive testing) from patients themselves. Radiological variables, such as tumor size and location, were assessed and recorded by the researchers to ensure uniformity of measurement. Clinician observed level of function was assessed using the Karnofsky Performance Score (KPS).

\section{Patient-reported outcome measures}

HRQoL was measured with the validated Short-Form Health Survey (SF-36), which yields 8 domain scores and two component scores [physical component summary (PCS) and mental component summary (MCS)], ranging from 0 to 100, with higher scores representing better HRQoL [16-18].

\section{Neuropsychological assessment}

Neuropsychological performance was assessed with a comprehensive battery of neuropsychological tests by trained research assistants and nurses: Digit-Symbol Substitution Test, Auditory Verbal Learning Test, Categoric Word Fluency Test, Concept Shifting Test, Memory Comparison Test, and Stroop Colour-Word Test [9, 10, 19]. Based on these tests, scores for the following neurocognitive domains were calculated: verbal memory, executive functioning, working 
memory, information processing speed, psychomotor functioning, and attention [9, 10, 19]. Each domain was transformed into $\mathrm{z}$-scores, using means and standard deviations from a reference sample from the Maastricht Aging Study (MAAS; large longitudinal study on the psychological and biological determinants of cognitive aging), matched on group-level for age, sex and educational level [20].

\section{Statistical analysis}

Multivariable regression analyses were performed to: (1) estimate the association between individual determinants, corrected for confounders, and impaired HRQoL and neurocognitive function, (2) build prediction models which could be used to predict the risk for impaired HRQoL or neurocognitive function for an individual patient based on patient-, tumor-, and treatment-related characteristics around diagnosis and intervention. Although for both analyses multivariable regression analyses are used, the statistical considerations and interpretation differ considerably. First, for the development of prediction models, only variables measured at baseline (around diagnosis and intervention) were included because the aim is to predict a future outcome. To assess determinants, variables later in the disease course were also considered (e.g. peritumoral edema before study assessment). Second, the outcomes of interest were dichotomized for the development of prediction models, as this facilitates use in clinical practice (i.e. does a patient have an impairment or not). For the analyses of determinants, outcomes were kept as continuous variables, as this increases statistical power.

Based on minimally clinically important differences as reported in the literature, HRQoL physical and mental component scores were dichotomized as follows: poor physical component score was defined as a score $<46.4$ and poor mental component score as a score $<47.0$ [21]. Impaired neurocognitive functioning was defined as a $\mathrm{z}$-score $<1.5$ in at least one out of six domains [22].

For all statistical tests, SPSS 23 (SPSS, Inc., Chicago, IL) was used, and p less than 0.05 was considered statistically significant.

\section{Analysis of determinants}

For the assessment of determinants, multivariable linear regression analyses were performed assessing the causal relationship between determinants (independent variable) and 5 outcomes (dependent variables): the SF-36 physical and mental component score (HRQoL), and z-scores for verbal memory, executive function, and attention (neurocognitive function). To reduce the number of analyses, only these 3/6 neurocognitive domains were chosen, as earlier analyses of this sample showed that patients primarily suffer from impairments in these domains [15]. Separate multivariable analyses were run for each association between a single determinant and a single outcome, corrected for possible confounders. A priori confounders were chosen for each analysis, based on prior knowledge and defined as associated with both the determinant and outcome, but not lying in the causal path between the determinant and outcome. Results were expressed as beta $(\beta)$ with $95 \%$ confidence intervals, CIs [14].

\section{Analysis of predictors}

For prediction analyses we developed two multivariable logistic regression models for the following two dichotomous outcomes (dependent variables): impaired HRQoL (physical component score $<46.4$ or mental component score $<47.0$ ) and impaired neurocognitive function ( $\mathrm{z}$-score $<1.5$ in at least one out of six domains). Based on the literature, clinically relevant variables were analyzed in univariable logistic regression analysis: gender, age, educational level, Charlson Comorbidity Index, tumor location and size, treatment characteristics (i.e. first resection, second resection, complications, radiotherapy), Simpson grading, WHO grade, years since diagnosis, and for the model predicting neurocognitive function also hand dominance. Variables were selected for multivariable analyses based on statistical significance in univariable regression analysis [3, 4, 7, 23-29]. A p $<0.20$ as selection criterion was used to limit chances of overfitting. Sensitivity analyses were performed with a cut-off of $\mathrm{p}<0.15$. We assessed the discrimination for each model, using the area under the receiver-operating curve (AUC) including $95 \%$ confidence interval (CI). For each model we provided two patient examples showing how to calculate the absolute risk of impaired HRQoL or neurocognitive function for an individual patient.

\section{Results}

A total of 190 patients (female: $n=149,78 \%$ ) were included in the analyses with a median follow-up since intervention of 9 years (IQR 7-12 years) (Table 1). Patients were on average 63 (SD 12) years old. Tumors were located on the skull base in 92 patients (48\%), the cerebral convexity in 93 patients (49\%) and other intracranial locations in 5 patients $(3 \%)$. The majority of operated patients were classified with a WHO grade I meningioma (88\%). Surgery was first line treatment in $168(88 \%)$ patients, $36(19 \%)$ received radiation.

A total of 93 (49\%) patients suffered from impaired HRQoL (PCS: $n=78,41 \%$; MCS $n=47,53 \%$ ), and 81 (43\%) from objective neurocognitive deficits. A total of 127 (67\%) suffered from a HRQoL impairment, or neurocognitive deficit. 
Table 1 Sociodemographic and clinical characteristics of the included meningioma patients

\begin{tabular}{|c|c|}
\hline & $\begin{array}{l}\text { Meningioma } \\
\text { patients } \\
\mathrm{n}=190\end{array}$ \\
\hline Age (years) & $63(\mathrm{SD} 12)$ \\
\hline Sex (female) & $149(78 \%)$ \\
\hline Academic hospital (vs. nonacademic teaching hospital) & $142(75 \%)$ \\
\hline \multicolumn{2}{|l|}{ Meningioma location } \\
\hline Skull base & $92(48 \%)$ \\
\hline Convexity & $93(49 \%)$ \\
\hline Other & $5(3 \%)$ \\
\hline Time since diagnosis (years) & $10(8-12)$ \\
\hline Tumor size before intervention (mm) & $38(26-50)$ \\
\hline Tumor size before study (mm) & $0(0-16)$ \\
\hline Tumor growth on last MRI before study & $10(5 \%)$ \\
\hline Number of meningiomas $\geq 2$ & $26(14 \%)$ \\
\hline Active MRI surveillance & $12(6 \%)$ \\
\hline Surgery as initial treatment & $168(88 \%)$ \\
\hline Complication first surgery (operated patients: $\mathrm{n}=168$ ) & $63(38 \%)$ \\
\hline Second surgery & $13(7 \%)$ \\
\hline Time since first surgery (years) & $9(7-12)$ \\
\hline \multicolumn{2}{|l|}{ Simpson grade (operated patients: $n=168$ ) } \\
\hline Grade I-III & $109(65 \%)$ \\
\hline Grade IV-V & $40(24 \%)$ \\
\hline Unknown & $19(11 \%)$ \\
\hline \multicolumn{2}{|l|}{ WHO grade (operated patients: $n=168$ ) } \\
\hline Grade I & $148(88 \%)$ \\
\hline Grade II & $12(7 \%)$ \\
\hline Unknown & $8(5 \%)$ \\
\hline Radiotherapy $^{\mathrm{a}}$ & $36(19 \%)$ \\
\hline Radiotherapy as initial treatment & $10(5 \%)$ \\
\hline Adjuvant radiotherapy & $26(14 \%)$ \\
\hline Time since radiotherapy (years) & $8(6-9)$ \\
\hline Karnofsky Performance Status at time of study & $100(90-100)$ \\
\hline Self-perceived neurocognitive impairment at time of study & $94(49 \%)$ \\
\hline Self-reported motor dysfunction at time of study & $55(29 \%)$ \\
\hline $\begin{array}{l}\text { Dexamethasone use for symptoms at any moment during the } \\
\text { care trajectory }\end{array}$ & $22(12 \%)$ \\
\hline Physical rehabilitation & $37(19 \%)$ \\
\hline Cognitive rehabilitation & $8(4 \%)$ \\
\hline Psychological support & $21(11 \%)$ \\
\hline Other supportive care & $10(5 \%)$ \\
\hline \multicolumn{2}{|l|}{ Educational level } \\
\hline Primary/secondary & $40(21 \%)$ \\
\hline Tertiary: technical/vocational & $85(45 \%)$ \\
\hline Academic & $59(31 \%)$ \\
\hline Not provided & $6(3 \%)$ \\
\hline \multicolumn{2}{|l|}{ Charlson Comorbidity Index } \\
\hline 0 & $127(67 \%)$ \\
\hline $1 \geq$ & $63(23 \%)$ \\
\hline Right handed & $147(77 \%)$ \\
\hline
\end{tabular}

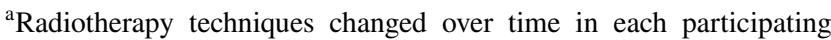
center, but all patients treated with radiotherapy received fractioned radiation

\section{Determinants}

\section{HRQoL}

Determinants for a lower physical component score (Table 2) were female sex (ref: male, $\beta=-2.52,95 \%$ CI -6.39 to 1.35 ), increase in Charlson Comorbidity Index ( $\beta=-3.31$ for each point increase, $95 \% \mathrm{CI}-4.62$ to -1.99$)$, larger tumor size before study participation $(\beta=-0.24,95 \% \mathrm{CI}-0.45$ to -0.02$)$, a lower educational level ( $\beta=2.70,95 \%$ CI 0.54 to 4.87$)$, and lower KPS ( $\beta=0.37,95 \%$ CI 0.17 to 0.58$)$. Determinant for a lower mental component score (Table 2) was lower KPS ( $\beta=0.39,95 \%$ CI 0.13 to 0.64 ). Tumor location, tumor size before intervention, surgical complications, reoperation, and radiotherapy were no determinants for HRQoL (Table 2).

\section{Neurocognitive function}

Determinants for decreased neurocognitive function (Table 3) for all three selected domains were radiotherapy (range $\beta-1.06$ to -0.47 ), second resection (range $\beta-2.34$ to -0.62 ), higher age (range $\beta-0.05$ to -0.03 ), and lower educational level (range $\beta$ for higher educational level: 0.31 to 0.91 ). Determinant for both decreased executive function and attention was lower KPS (range $\beta 0.06$ to 0.07$)$. Determinants for worse executive function were maximum tumor size $(\beta=-0.03$ for each $\mathrm{mm}$ tumor, $95 \% \mathrm{CI}-0.05$ to -0.01 ) and edema on the last MRI before study participation (ref: no edema $\beta=-0.84$, $95 \% \mathrm{CI}-1.70$ to -0.01$)$. Determinant for decreased attention was complications of first resection $(\beta=-0.76,95 \%$ CI -1.42 to -0.10). Tumor location, tumor size before intervention were no determinants for neurocognitive function (Table 3 ).

\section{Prediction models}

\section{HRQoL impairments}

Using a p-value cut-off $<0.20$ in univariable analyses, the following variables were included in the multivariable prediction model: age, tumor size before intervention, surgery, surgical complications, Charlson Comorbidity Index and educational level (Table 4). This model showed an AUC of 0.72 (95\% CI 0.63 to 0.80 ) (Supplementary Fig. 1). Sensitivity analysis resulted in a model with the same variables, except for age, with also a similar AUC of 0.72. The full prediction model to calculate the absolute 
Table 2 Determinants for Health-related Quality of Life as measured with the Short-from 36 (SF-36), separately for the physical and mental component score

\begin{tabular}{|c|c|c|}
\hline & Physical component score $\beta$ (95\% CI) & Mental component score $\beta(95 \% \mathrm{CI})$ \\
\hline Sex female (ref: male) & $-2.521(-6.393$ to 1.351$)$ & $0.066(-4.182$ to 4.315$)$ \\
\hline Age (years) & $-0.113(-0.248$ to 0.023$)$ & $-0.016(-0.165$ to 0.133$)$ \\
\hline Tumor location, skull base (ref: convexity) & $2.832(-0.410$ to 6.073$)$ & $2.603(-0.974$ to 6.180$)$ \\
\hline Tumor size before last intervention (mm) & $0.085(-0.017$ to 0.187$)$ & $0.023(-0.086$ to 0.132$)$ \\
\hline Tumor size before study (mm) & $-0.235(-0.450$ to -0.020$)$ & $0.20(-0.211$ to 0.252$)$ \\
\hline Tumor growth on last MRI before study, yes (no) & $0.571(-1.479$ to 2.626$)$ & $0.816(-1.396$ to 3.029$)$ \\
\hline Edema on last MRI before study, yes (ref: no) & $-2.798(-10.988$ to 5.392$)$ & $4.801(-4.077$ to 13.678$)$ \\
\hline First resection, yes (ref: no) & $1.438(-5.564$ to 8.439$)$ & $3.072(-4.852$ to 10.997$)$ \\
\hline First resection complications, yes (ref: no) & $-1.873(-5.596$ to 1.851$)$ & $-0.444(-4.648$ to 3.760$)$ \\
\hline Second resection, yes (ref: no) & $-1.325(-8.290$ to 5.640$)$ & $1.610(-6.590$ to 9.811$)$ \\
\hline Simpson grade first resection IV/V (ref: I-III) & $-1.241(-3.001$ to 0.519$)$ & $1.693(-0.216$ to 3.602$)$ \\
\hline WHO Grade II (ref: I) & $-0.027(-6.657$ to 6.603$)$ & $-4.843(-11.988$ to 2.301$)$ \\
\hline Radiotherapy, yes (ref: no) & $-2.950(-7.837$ to 1.936$)$ & $-3.327(-9.083$ to 2.429$)$ \\
\hline Karnofsky performance score & $0.374(0.170$ to 0.578$)$ & $0.388(0.133$ to 0.643$)$ \\
\hline Hand dominance, right (ref: left) & $-3.117(-7.694$ to 1.460$)$ & $1.168(-3.815$ to 6.152$)$ \\
\hline Charlson Comorbidity Index & $-3.308(-4.624$ to -1.992$)$ & $-0.021(-1.560$ to 1.517$)$ \\
\hline $\begin{array}{l}\text { Educational level (1: primary/secondary, 2: tertiary voca- } \\
\text { tional, 3: academic) }\end{array}$ & $2.703(0.540$ to 4.867$)$ & $0.762(-3.512$ to 5.036$)$ \\
\hline Years since diagnosis & $-0.460(-0.500$ to 0.410$)$ & $-0.090(0.720$ to 0.400$)$ \\
\hline
\end{tabular}

Results marked in bold are significant

$\beta$ Represent the decrease or increase in physical or mental component score. For continuous determinants this is per 1-point increase in the determinant, unless otherwise specified. For categorical variables a comparison is made with a reference category

Table 3 Determinants for neurocognitive functioning as measured with a standardized test battery for the three previously determined most relevant domains in this patient population

\begin{tabular}{|c|c|c|c|}
\hline & Verbal memory $\beta$ (95\% CI) & Executive function $\beta(95 \% \mathrm{CI})$ & Attention $\beta(95 \% \mathrm{CI})$ \\
\hline Sex female (ref: male) & $0.442(0.140$ to 0.744$)$ & $0.107(-0.332$ to 0.546$)$ & $0.341(-0.350$ to 1.032$)$ \\
\hline Age (years) & $-0.025(-0.036$ to -0.014$)$ & $-0.048(-0.063$ to -0.032$)$ & $-0.042(-0.067$ to -0.018$)$ \\
\hline Tumor location, skull base (ref: convexity) & $0.034(-0.223$ to 0.290$)$ & $-0.122(-0.499$ to 0.255$)$ & $0.010(-0.588$ to 0.608$)$ \\
\hline Tumor size before last intervention (mm) & $-0.004(-0.012$ to 0.004$)$ & $-0.005(-0.015$ to 0.006$)$ & $-0.004(-0.022$ to 0.014$)$ \\
\hline Tumor size before study (mm) & $-0.007(-0.023$ to 0.009$)$ & $-0.028(-0.051$ to -0.005$)$ & $-0.024(-0.065$ to 0.016$)$ \\
\hline $\begin{array}{l}\text { Tumor growth on last MRI before study, yes (ref: } \\
\text { no) }\end{array}$ & $-0.250(-1.336$ to 0.836$)$ & $0.019(-0.194$ to 0.231$)$ & $0.170(-0.181$ to 0.521$)$ \\
\hline Edema on last MRI before study, yes (ref: no) & $-0.281(-0.892$ to 0.330$)$ & $-0.844(-1.701$ to -0.014$)$ & $-0.605(-2.023$ to 0.813$)$ \\
\hline First resection, yes (ref: no) & $0.693(0.130$ to 1.256$)$ & $-0.069(-0.850$ to 0.714$)$ & $-0.279(-1.606$ to 1.048$)$ \\
\hline First resection complications, yes (ref: no) & $-0.228(-0.553$ to 0.097$)$ & $-0.357(-0.761$ to 0.047$)$ & $-0.758(-1.415$ to -0.101$)$ \\
\hline Second resection, yes (ref: no) & $-0.623(-1.188$ to -0.057$)$ & $-1.025(-1.815$ to -0.236$)$ & $-2.336(-3.680$ to -0.993$)$ \\
\hline Simpson grade first resection IV/V (ref: I-III) & $0.094(-0.040$ to 0.229$)$ & $0.040(-0.147$ to 0.227$)$ & $0.163(-0.167$ to 0.492$)$ \\
\hline WHO Grade II (ref: I) & $0.372(-0.104$ to 0.847$)$ & $0.185(-0.530$ to 0.899$)$ & $0.038(-1.108$ to 1.184$)$ \\
\hline Radiotherapy, yes (ref: no) & $-0.469(-0.866$ to -0.071$)$ & $-0.666(-1.224$ to -0.107$)$ & $-1.063(-2.036$ to -0.090$)$ \\
\hline Karnofsky performance score & $0.012(-0.006$ to 0.030$)$ & $0.060(0.035$ to 0.085$)$ & $0.069(0.025$ to 0.113$)$ \\
\hline Hand dominance, right (ref: left) & $0.520(0.167$ to 0.873$)$ & $0.359(-0.168$ to 0.886$)$ & $0.213(-0.693$ to 1.065$)$ \\
\hline Charlson Comorbidity Index & $-0.019(-0.128$ to 0.090$)$ & $-0.125(-0.285$ to 0.036$)$ & $-0.133(-0.383$ to 0.116$)$ \\
\hline $\begin{array}{l}\text { Educational level (1: primary/secondary, } 2 \text { : ter- } \\
\text { tiary vocational, } 3 \text { : academic) }\end{array}$ & $0.305(0.139$ to 0.471$)$ & $0.510(0.265$ to 0.756$)$ & $0.913(0.528$ to 1.297$)$ \\
\hline Years since diagnosis & $-0.007(-0.045$ to 0.031$)$ & $-0.033(-0.090$ to 0.023$)$ & $-0.06(-0.140$ to 0.030$)$ \\
\hline
\end{tabular}

Results marked in bold are significant

$\beta$ Represent the decrease or increase in z-score. For continuous determinants this is per 1-point increase in the determinant, unless otherwise specified. For categorical variables a comparison is made with a reference category 
Table 4 Prediction model development for impaired Health-related quality of life

Univariable analysis odds ratio (95\% CI)

Multivariable model based on statistical significance only odds ratio $(95 \%$ CI)

Sex female (ref: male)
Age (years)
Tumor location, skull base (ref: convexity)
Tumor size before last intervention (mm)
First resection yes (ref: no)
First resection complications yes (ref: no)
Second resection yes (ref: no)
Simpson grade first resection IV/V (ref: I-III)
WHO Grade II (ref: I)
Radiotherapy yes (ref: no)
Charlson Comorbidity Index
Educational level (1: primary/secondary, 2: tertiary
vocational, 3: academic)
Years since diagnosis
1.024 (0.505 to 2.076), $\mathrm{p}=0.947$
1.018 ( 0.992 to 1.044$), \mathrm{p}=0.173$
0.997 (0.964 to 1.030$)$
0.801 ( 0.446 to 1.437$), \mathrm{p}=0.456$
0.982 (0.964 to 1.001$), p=0.061$
$0.980(0.959$ to 1.002$)$
0.408 ( 0.158 to 1.052$), \mathrm{p}=0.064$
0.438 ( 0.117 to 1.637$)$
2.066 (1.102 to 3.873$), \mathrm{p}=0.024$
1.924 (0.900 to 4.114$)$
1.406 ( 0.411 to 4.804$), \mathrm{p}=0.587$
1.502 ( 0.724 to 3.118$), p=0.275$
1.772 ( 0.537 to 5.845$), \mathrm{p}=0.348$
$1.610(0.575$ to 3.421$), \mathrm{p}=0.216$
1.520 (1.117 to 2.069$), \mathrm{p}=0.008$
1.338 ( 0.930 to 1.925$)$
0.535 ( 0.351 to 0.816$), p=0.004$
$0.428(0.255$ to 0.717$)$
$1.036(0.953$ to 1.127$), p=0.406$

Health-related quality of life impairment is defined as a physical component score $<46.4$ or mental component score $<47.0$ ). p-values are only showed for the univariable analysis, as they were used for development of the multivariable model that was based on statistical significance

risk of impaired HRQoL is presented in Supplementary Table 1 .

\section{Neurocognitive impairments}

Using a p-value cut-off $<0.20$ in univariable analyses, the following variables were included in the multivariable prediction model: age, tumor size before intervention, reresection, radiotherapy, educational level, and years since diagnosis (Table 5). This model showed an AUC of 0.78 (95\% CI 0.70 to 0.85 ) (Supplementary Fig. 1). Sensitivity analysis resulted in the same model with the same variables and hence the same AUC. The full prediction model to calculate the absolute risk of impaired neurocognitive function is presented in Supplementary Table 2.

Table 5 Prediction model development for Neurocognitive deficits

Univariable analysis odds ratios $(95 \% \mathrm{CI})$
Multivariable model based on statistical significance odds ratios $(95 \% \mathrm{CI})$

Gender female (ref: male)

1.089 ( 0.540 to 2.196$), \mathrm{p}=0.813$

1.036 (1.008 to 1.064$), \mathrm{p}=0.011$

1.024 (0.987 to 1.063$)$

1.072 (0.598 to 1.923$), \mathrm{p}=0.816$

1.019 (1.000 to 1.039$), \mathrm{p}=.048$

1.022 (0.998 to 1.047$)$

0.729 (0.299 to 1.777$), \mathrm{p}=0.487$

1.500 ( 0.805 to 2.794$), p=0.201$

4.574 (1.191 to 17.572$), p=0.027$

2.662 (0.488 to 14.528$)$

1.121 ( 0.540 to 2.325$), p=0.760$

2.148 ( 0.651 to 7.092 ), $p=0.210$

2.011 ( 0.956 to 4.230$), p=0.066$

0.659 ( 0.289 to 1.505$), p=0.323$

1.135 ( 0.877 to 1.468$), p=0.336$

0.412 ( 0.265 to 0.641$), p=0.000$

0.359 (0.206 to 0.628$)$

1.103 (1.011 to 1.203$), \mathrm{p}=0.027$
$1.130(0.982$ to 1.301$)$
Years since diagnosis

Neurocognitive deficit is defined as a $\mathrm{z}$-score $<1.5$ in at least one neurocognitive domain. p-values are only showed for the univariable analysis, as they were used for development of the multivariable models that was based on statistical significance 


\section{Examples}

Example patients and calculations are provided for both prediction models in Supplementary Tables 1 and 2. Furthermore, using the predicted risk for HRQoL impairment, the sample was divided into tertiles (i.e. three equally large groups: low-risk, medium-risk, high-risk). Of the patients in the low-risk group 27\% suffered an HRQoL impairment, $40 \%$ in the medium-risk group, and $70 \%$ in the high-risk group. Using the predicted risk for neurocognitive impairment to divide patients in risk groups, $9 \%$ of patients in the low-risk group suffered from a neurocognitive impairment, $47 \%$ in the medium-risk group, and $60 \%$ of patients in the high-risk group.

\section{Discussion}

Results of this study indicate that determinants for the longterm disease burden in meningioma patients on group level are (1) sociodemographic characteristics: sex, age and educational level, (2) treatment characteristics: complications of surgery, reoperation, radiotherapy, (3) tumor characteristics: diameter and peritumoral edema at the time of study, and (4) clinician-reported level of functioning (i.e. KPS). Furthermore, we have developed prediction models to predict whether an individual patient will suffer from long-term HRQoL or neurocognitive impairment using easily accessible patient chart information, which showed moderate to good discriminative ability to differentiate between those with and without clinically relevant impairments in HRQoL or neurocognitive function on the long term. We reported that $67 \%$ of patients suffered from impaired HRQoL or neurocognitive deficits. For these patients, rehabilitation and supportive care options should be available, even on the long-term, as the need for these supportive treatments was underlined in a previous study in meningioma patients [30]. In this study we focused on readily available variables as determinants and predictors, facilitating use in daily clinical practice.

\section{Interpretation: meningioma literature on determinants for disease burden}

Information on determinants might be useful for clinicians to better understand the impact of both the tumor and treatment on the long-term outcomes of patients. We report that a complicated treatment course with surgical complications, the need for reoperation and radiotherapy, are associated with long-term neurocognitive impairments and less with HRQoL impairments, which is in line with the literature on (low grade) glioma patients [9, 31]. On a group-level, meningioma patients therefore deserve extra attention regarding neurocognitive deficits and early referral for neurocognitive rehabilitation. Furthermore, results of this study showed that tumor activity at the time of study, defined as the presence of edema and a larger tumor diameter on the last MRI before study participation, were negatively associated with patients' executive function. A larger tumor diameter was also associated with decreased physical function. This is in line with previous meningioma studies reporting in the first years after treatment that factors negatively influencing overall HRQoL and neurocognitive function were higher histological grade, a larger tumor size and peritumoral edema [23, $25,32]$. However, we found no association between WHO grade and HRQoL or neurocognitive function, which might be explained by the low number of patients with WHO grade II tumors in our study (7\%). Indeed, based on the WHO 2016 classification of central nervous system tumors, WHO grade II tumors occur in up to $20 \%$ of patients [1]. Our results may therefore not be completely generalizable, as we have a slight underrepresentation of patients with WHO grade II tumors. Two previous studies reported, using univariable analyses only, that tumor location and tumor laterality were associated with neurocognitive function, while in the current study no association was observed after correction for confounders [7, 25, 27, 28]. These results have implications for our understanding of the disease burden in meningioma, as generally it is thought that patients with skull base lesions, compared with convexity tumors, suffer from worse HRQoL after surgery [3].

\section{Interpretation: prediction models for individual meningioma patients}

Prediction models were developed to estimate which patient develops a long-term impairment in HRQoL or neurocognitive function. Until now there have been no prediction models developed for the short- or long-term disease burden of meningioma patients. Not only does the disease burden changes over time, as HRQoL and neurocognitive impairments become more prominent after 5 years of follow-up $[3-5,15,29]$. It has also been acknowledged that patients enter a chronic disease state in the long-term, with specific long-term survivor issues [3, 4]. With good survival rates of this patient population, a prediction model for the long-term disease burden is of particular interest. Two separate models were built, one for long-term problems in HRQoL and one for neurocognitive impairments. These models showed that higher age, lower educational level, presence of comorbidities as measured with the Charlson Comorbidity Index, larger tumor size before intervention, surgical complications, the need for reresection, initiation of radiotherapy, and years since diagnosis were predictors for long-term impairments. Although these variables together help to predict these future outcomes, not all of these variables were independently 
related to the measured outcomes (i.e. determinants), such as tumor location. This emphasizes the difference between predictors and determinants. While determinants are variables causally related to the outcome of interest, predictors are solely used to predict the outcome of interest, without assuming causality. Hence, predictors can be determinants, act as a proxy for a determinant, or have no causal relationship at all with the long-term disease burden.

\section{Limitations}

The measured outcomes in this study are 9 years after the last intervention. Therefore, the studied patients might have experienced other major health issues and undergone large extracranial treatments between the period of meningioma treatment and study participation, which could impact their long-term HRQoL and neurocognitive function. Furthermore, a limitation of the current study is the lack of external validation of the models. Prediction models that are only internally validated might be overfitted with externally validated models showing lower performance measures. This might especially hold true for the models predicting HRQoL, as it is strongly subjected to the sociocultural context and different health care systems. Cross-cultural validation is therefore warranted. Furthermore, due to the cross-sectional nature of our study, we were unable to assess determinants and predictors for a change in HRQoL or neurocognitive function over time. Previous studies have shown that baseline HRQoL also acts as predictor for long-term HRQoL, which is a more precise measure of functioning than the KPS [3]. In the light of lack of a validated meningioma-specific HRQoL instrument, we used the SF-36 to measure HRQoL, as this is the most frequently used HRQoL instrument in meningioma literature and in other diseases, facilitating comparability of our results [3]. However, HRQoL issues specific to this patient group might therefore be missing [2]. Previous research has indeed shown that existing HRQoL questionnaires currently used in meningioma patients do not fully cover all relevant issues, supporting the need to develop and validate a meningioma-specific HRQoL questionnaire.

\section{Implications for clinical practice}

The found determinants can help clinicians to better understand the long-term HRQoL and neurocognitive impairments of patients, as both the impact of the tumor and the treatment they initiate may affect patients' functioning and well-being. The prediction models can be used to identify individual patients at baseline with a high risk to suffer from a long-term disease burden, which enables tailored provision of information and allocation of scarce supportive care services to those most likely to obtain benefit. Our results emphasize that predictors are not per se determinants, and that causal attributions shouldn't be given to predictors. We recommend external validation in the country of the population of interest before clinical use of the described prediction models.

Acknowledgements We would like to acknowledge the research nurses of the LUMC/HMC Neurosurgery Department for their help with the data collection.

The generation of this manuscript has been supported by the Dutch Meningioma Consortium which at time of submission of this manuscript consisted of Florien W. Boele, Linda Dirven, Wouter R. van Furth, Martin Klein, Johan Koekkoek, Frank Lagerwaard, Pim B. van der Meer, Saskia M. Peerdeman, Jaap C. Reijneveld, Martin J.B. Taphoorn, Amir H. Zamanipoor Najafabadi. Other collaborators of the Dutch Meningioma Consortium: Wouter A. Moojen, Jaap C. Reijneveld.

Author contributions LD, FWB, and SM designed the original study of which data was used for this report. Data collection was performed by AHZN and PBvdM. AHZN initiated the assessment of the reported study questions and performed data analysis with input from LD. AHZN wrote the first and successive versions of the manuscript. All authors contributed to the interpretation of the results, intellectual content, critical revisions to the drafts of the paper, and approved the final version. LD supervised the project. AHZN had full access to all the data in the study and had final responsibility for the decision to submit for publication.

Funding AHZN was supported by a personal MD/PhD Grant of the Leiden University Medical Center. No specific funding was received for this project.

Data availability Upon request (please direct to amir@lumc.nl) the used code for the analysis can be provided.

Code availability Upon request (please direct to amir@lumc.nl) the used code for the analysis can be provided.

\section{Compliance with ethical standards}

Conflict of interest None of the authors declares a conflict of interest.

Consent for publication All authors have reviewed and given consent to this submission of this manuscript.

Ethical approval This cross-sectional study was approved by the Medical Ethical Committees of all participating centers (NL54866.029.15).

Informed consent Participants provided informed consent before study procedures.

Open Access This article is licensed under a Creative Commons Attribution 4.0 International License, which permits use, sharing, adaptation, distribution and reproduction in any medium or format, as long as you give appropriate credit to the original author(s) and the source, provide a link to the Creative Commons licence, and indicate if changes were made. The images or other third party material in this article are included in the article's Creative Commons licence, unless indicated otherwise in a credit line to the material. If material is not included in the article's Creative Commons licence and your intended use is not permitted by statutory regulation or exceeds the permitted use, you will 
need to obtain permission directly from the copyright holder. To view a copy of this licence, visit http://creativecommons.org/licenses/by/4.0/.

\section{References}

1. Ostrom QT, Gittleman H, Truitt G et al (2018) CBTRUS Statistical Report: primary brain and other central nervous system tumors diagnosed in the United States in 2011-2015. Neuro-oncology 20:1-86. https://doi.org/10.1093/neuonc/noy131

2. Zamanipoor Najafabadi AH, Peeters MCM, Lobatto DJ et al (2017a) Health-related quality of life of cranial WHO grade I meningioma patients: are current questionnaires relevant? Acta Neurochir (Wien) 159:2149-2159. https://doi.org/10.1007/s0070 1-017-3332-8

3. Zamanipoor Najafabadi AH, Peeters MCM, Lobatto DJ et al (2017b) Impaired health-related quality of life of meningioma patients-a systematic review. Neuro-oncology 19(7):897-907. https://doi.org/10.1093/neuonc/now250

4. Nassiri F, Price B, Shehab A et al (2019) Life after surgical resection of a meningioma: a prospective cross-sectional study evaluating health-related quality of life. Neuro-oncology 21:I32-I43. https://doi.org/10.1093/neuonc/noy152

5. Meskal I, Gehring K, Rutten GJM, Sitskoorn MM (2016) Cognitive functioning in meningioma patients: a systematic review. J Neurooncol 128:195-205

6. Rijnen S, Meskal I, Bakker M et al (2018) Cognitive outcomes in meningioma patients undergoing surgery: individual changes over time and predictors of late cognitive functioning. Neuro-oncology 20:1-12. https://doi.org/10.1093/neuonc/noy139.387

7. Dijkstra M, van Nieuwenhuizen D, Stalpers LJ et al (2009) Late neurocognitive sequelae in patients with WHO grade I meningioma. J Neurol Neurosurg Psychiatry 80:910-915

8. Bosma I, Reijneveld JC, Douw L et al (2009) Health-related quality of life of long-term high-grade glioma survivors. Neurooncology 11:51-58. https://doi.org/10.1215/15228517-2008-049

9. Douw L, Klein M, Fagel SS et al (2009) Cognitive and radiological effects of radiotherapy in patients with low-grade glioma: long-term follow-up. Lancet Neurol 8:810-818. https://doi. org/10.1016/S1474-4422(09)70204-2

10. Klein M, Heimans JJ, Aaronson NK et al (2002) Effect of radiotherapy and other treatment-related factors on mid-term to longterm cognitive sequelae in low-grade gliomas: a comparative study. Lancet 360:1361-1368. https://doi.org/10.1016/S0140 $-6736(02) 11398-5$

11. Lovely MP, Stewart-Amidei C, Page M et al (2013) A new reality: long-term survivorship with a malignant brain tumor. Oncol Nurs Forum 40:267-274. https://doi.org/10.1188/13.ONF.267-274

12. Salander P, Bergenheim T, Henriksson R (1996) The creation of protection and hope in patients with malignant brain tumours. Soc Sci Med 42:985-996. https://doi.org/10.1016/02779536(95)00204-9

13. Brown CB, Leeper HE (2018) The role of survivorship care for patients with glioma. Semin Oncol Nurs 34:547-552. https://doi. org/10.1016/j.soncn.2018.10.015

14. Van Diepen M, Ramspek CL, Jager KJ et al (2017) Prediction versus aetiology: common pitfalls and how to avoid them. Nephrol Dial Transplant 32:ii1-ii5. https://doi.org/10.1093/ndt/gfw459

15. Zamanipoor Najafabadi AH, van der Meer PB, Boele FW et al (2020) Long-term disease burden and survivorship issues after surgery and radiotherapy of intracranial meningioma patients. Neurosurgery. https://doi.org/10.1093/neuros/nyaa351
16. Ware JEJ, Sherbourne CD (1992) The MOS 36-Item Short-Form Health Survey (SF-36) I. Conceptual framework and item selection. Med Care 30:473-483

17. McHorney CA, Ware JEJ, Raczek AE (1993) The MOS 36-item Short-Form Health Survey (SF-36): II. Psychometric and clinical test of validity in measuring physical and mental health constructs. Med Care 31:247-263

18. Brazier JE, Harper R, Jones NM et al (1992) Validating the SF-36 health survey questionnaire: new outcome measure for primary care. BMJ 305:160-164. https://doi.org/10.1136/ bmj.305.6846.160

19. Ediebah DE, Reijneveld JC, Taphoorn MJB et al (2017) Impact of neurocognitive deficits on patient-proxy agreement regarding health-related quality of life in low-grade glioma patients. Qual Life Res 26:869-880. https://doi.org/10.1007/s11136-016-1426-z

20. van Boxtel MPJ, Buntinx F, Houx PJ et al (1998) The relation between morbidity and cognitive performance in a normal aging population. J Gerontol 53A:M147-M155

21. Edelvik A, Taft C, Ekstedt G, Malmgren K (2017) Health-related quality of life and emotional well-being after epilepsy surgery: a prospective, controlled, long-term follow-up. Epilepsia 58:17061715. https://doi.org/10.1111/epi.13874

22. Lezak MD, Howieson DB, Loring DW (2004) Neuropsychological assessment. Oxford University Press, New York

23. Mohsenipour I, Deusch E, Gabl M et al (2001) Quality of life in patients after meningioma resection. Acta Neurochir 143:547-553

24. Miao Y, Qiu Y, Lin Y, Lu X (2008) Assessment of self-reported and health-related quality of life in patients with brain tumours using a modified questionnaire. J Int Med Res 36:1279-1286

25. Liouta E, Koutsarnakis C, Liakos F, Stranjalis G (2016) Effects of intracranial meningioma location, size, and surgery on neurocognitive functions: a 3-year prospective study. J Neurosurg 124:1578-1584. https://doi.org/10.3171/2015.6.JNS1549.1578

26. Waagemans ML, Van Nieuwenhuizen D, Dijkstra M et al (2011) Long-term impact of cognitive deficits and epilepsy on quality of life in patients with low-grade meningiomas. Neurosurgery 69:72-78. https://doi.org/10.1227/NEU.0b013e318212badb

27. Kangas M, Williams JR, Smee RI (2012) The association between post-traumatic stress and health-related quality of life in adults treated for a benign meningioma. Appl Res Qual Life 7:163-182

28. Mathiesen T, Gerlich $\AA$, Kihlström L et al (2007) Effects of using combined transpetrosal surgical approaches to treat petroclival meningiomas. Neurosurgery 60:982-991. https://doi. org/10.1227/01.NEU.0000255476.06247.F1

29. Rijnen SJM, Meskal I, Bakker M et al (2019) Cognitive outcomes in meningioma patients undergoing surgery: individual changes over time and predictors of late cognitive functioning. Neurooncology 21:911-922. https://doi.org/10.1093/neuonc/noz039

30. Zamanipoor Najafabadi AH, van de Mortel JPM, Lobatto DJ et al (2020) Unmet needs and recommendations to improve meningioma care through patient, partner, and health care provider input: a mixed-method study. Neuro-oncol Pract 7:239-248. https://doi. org/10.1093/nop/npz055

31. Klein M, Duffau H, De Witt Hamer PC (2012) Cognition and resective surgery for diffuse infiltrative glioma: an overview. J Neurooncol 108:309-318

32. Miao Y, Lu X, Qiu Y et al (2010) A multivariate analysis of prognostic factors for health-related quality of life in patients with surgically managed meningioma. J Clin Neurosci 17:446-449

Publisher's Note Springer Nature remains neutral with regard to jurisdictional claims in published maps and institutional affiliations.

Prior presentations The results described in this manuscript have not been presented at any conferences. 


\section{Affiliations}

Amir H. Zamanipoor Najafabadi' 1,2,3 (10 - Pim B. van der Meer ${ }^{3} \cdot$ Florien W. Boele $^{4,5} \cdot$ Martin J. B. Taphoorn $^{3,7}$. Martin Klein $^{8}$. Saskia M. Peerdeman ${ }^{6} \cdot$ Wouter R. van Furth $^{1,2} \cdot$ Linda Dirven $^{3,7}$

1 Department of Neurosurgery, University Neurosurgical Center Holland, Leiden University Medical Center, Albinusdreef 2, Postal Zone J11-R, 2333ZA Leiden, The Netherlands

2 Haaglanden Medical Center \& Haga Teaching Hospitals, The Hague, The Netherlands

3 Department of Neurology, Leiden University Medical Center, Albinusdreef 2, Postal Zone J11-R, 2333ZA Leiden, The Netherlands

4 Leeds Institute of Medical Research at St James's, St James's University Hospital, Leeds LS9 7TF, UK
5 Faculty of Medicine and Health, Leeds Institute of Health Sciences, University of Leeds, Leeds LS2 9JT, UK

6 Department of Neurosurgery, Amsterdam UMC, Vrije Universiteit Amsterdam, Amsterdam, The Netherlands

7 Department of Neurology, Haaglanden Medical Center, The Hague, The Netherlands

8 Department of Medical Psychology, Brain Tumor Center Amsterdam, Amsterdam UMC, Vrije Universiteit Amsterdam, Amsterdam, The Netherlands 